\title{
Vie et nature dans la phénoménologie contemporaine : vers un enrichissement du naturalisme
}

Par NICOLAS ZASLAWSKI

Université de Lausanne, Université Jean Moulin Lyon 3

\begin{abstract}
Prenant acte des développements les plus récents de la tradition phénoménologique, du côté des sciences cognitives d'une part, et du côté de la vie, d'autre part, le présent article se propose d'évaluer à quelles conditions ces développements sont susceptibles d'informer la phénoménologie sur ses propres «pré-conditions». Montrant comment le projet dit de « naturalisation » de la phénoménologie et la phénoménologie de la vie dans sa version barbarassienne convergent en pointant une même insuffisance de l'effort phénoménologique traditionnel, l'auteur propose, à partir de l'accointance philosophique, étonnante au premier abord, de ces deux courants de pensée, d'indiquer comment la phénoménologie peut espérer achever l'enquête sur ses propres "pré-conditions » en s'intégrant le cognitif naturaliste au moyen d'une conception réévaluée de la nature elle-même.
\end{abstract}

\section{Introduction : vie, nature et phénoménologie au $\mathrm{XXI}^{\mathrm{e}}$ siècle}

Dans un article consacré à Jan Patočka, Ovidiu Stanciu relevait avec lui le risque d'«enlisement subjectiviste » inhérent à la phénoménologie : celle-ci s'est toujours trouvée tentée «d'interpréter sa propre découverte l'apparaître comme tel, le champ phénoménal - comme une structure subjective ${ }^{1}$.» Afin de pallier cette difficulté, il constate, avec Patočka, l'exigence de «fournir une fondation » à la phénoménologie, seul moyen de

\footnotetext{
${ }^{1}$ Ovidiu Stanciu (2017). De la manifestation au réel. La phénoménologie asubjective et son autodépassement. Revue de métaphysique et de morale, 3 (95), p. 304.
} 
conférer à celle-ci de quoi « rendre raison de sa propre possibilité ${ }^{1}$ ». Ce geste, relève Stanciu, prend pour point d'appui, chez Patočka, le problème « de l'inscription du champ phénoménal dans la physis ${ }^{2}$. » Il conclut alors en remarquant que «ces démarches [...] visent seulement à suppléer à la recherche phénoménologique, en fournissant un éclaircissement sur ses préconditions ${ }^{3} »$.

Il ne s'agit pas pour nous d'interroger ici la phénoménologie patočkienne ni même de discuter les analyses conduites par Stanciu à son propos ; en revanche, ces remarques préliminaires doivent continuer de nous préoccuper en raison de leur portée indéniable pour la phénoménologie en général, au-delà de l'œuvre de Patočka. Ces conclusions aboutissent en effet à un système de problèmes auxquels la phénoménologie n'a que récemment ${ }^{4}$ commencé à se confronter et qu'elle ne pourra plus éluder. Il nous importe à ce titre d'en souligner l'importance et de nous inscrire dans leur continuité. Les questions soulevées par l'analyse du travail de Patočka par Stanciu rejoignent en effet les préoccupations centrales du débat phénoménologique contemporain : là où il dépeint la phénoménologie comme «contrainte de ménager une place pour une ontologie, sans laquelle elle serait suspendue dans le vide ${ }^{5}$ », Jean-Luc Petit, dans un "bilan» des débats consacrés à la « naturalisation de la phénoménologie », interrogeait quant à lui le risque, encouru par le « sujet transcendantal », de «lévitation par rapport au substrat empirique $^{6} »$; là où Stanciu pointe, à travers Patočka, la nécessité d'une « fondation » pour la phénoménologie, Renaud Barbaras soulignait l'importance d'une «fondation authentique de la conscience dans la vie ${ }^{7}$ » que la phénoménologie aurait selon lui toujours manquée en raison de ses "présupposés » intellectualistes; là, enfin, où Stanciu rencontre avec Patočka la question d'une "physis [...] dans sa connexion (de fondation)

\footnotetext{
${ }^{1}$ Ibid., p. 315.

${ }^{2}$ Ibid., p. 313.

${ }^{3}$ Ibid., p. 316.

${ }^{4}$ Stanciu a esquissé une analyse en ce sens dans une communication sur le « tournant cosmologique » de la phénoménologie à laquelle nous avons assisté lors de l'atelier "phénoménologies contemporaines» du Center for Subjectivity Research de Copenhague le 08 mars 2017.

${ }^{5}$ Stanciu, art. cit., p. 314 (nous soulignons).

${ }^{6}$ Jean-Luc Petit (2015). Présentation. Les Cahiers Philosophiques de Strasbourg, (38), p. 13 (nous soulignons).

${ }^{7}$ Renaud Barbaras (2008). Introduction à une phénoménologie de la vie. Paris : Vrin, p. 232, désormais abrégé $I P V$.
} 
avec le champ phénoménal ${ }^{1}$ », Dan Zahavi soulignait la nécessité « d'opérer avec une notion plus riche de nature ${ }^{2} »$ alors que Barbaras poursuivait son effort dans le vital en l'inscrivant dans la pensée d'une « $\varphi v ́ \sigma l \varsigma$, conçue comme le procès du devenir de la nature ${ }^{3} »$.

Dans La philosophie en France au $X X^{e}$ siècle, Frédéric Worms nous offre par ailleurs le moyen de préciser ces convergences. En effet, son « concept [...] de moment philosophique ${ }^{4} »$ est présenté par Worms comme l'instrument méthodologique d'une appréhension sensée du déploiement temporel des doctrines philosophiques ${ }^{5}$, sachant que le «moment» se définit par des « relations, ouvertes, tendues, entre des œuvres singulières ${ }^{6}$ » et qu'il présente également les caractéristiques de la distinction et de la cohérence, celles-ci étant assurées par l'articulation « des œuvres singulières [...] autour de problèmes communs ${ }^{8} »$. Proposant, à l'issue de son livre, une interrogation de notre modernité philosophique à l'aune de sa pensée du moment, Worms est singulièrement conduit à «parler de notre moment, en un sens fort, comme du moment du vivant ${ }^{9} »$. Or de manière tout à fait significative, il invoque à l'appui d'une telle affirmation «l'extension [contemporaine] $d u$ problème ou du modèle du vivant à tous les domaines du savoir et de la pratique », extension dont les composantes comportent, entre autres, " des recherches originales confrontant "phénoménologie et naturalisation", élaborant de nouvelles phénoménologies de la vie ${ }^{10} »$. En d'autres termes, si l'on suit Worms, les principes philosophiques guidant à la fois le projet de

\footnotetext{
${ }^{1}$ Stanciu, art. cit., p. 316.

${ }^{2}$ Dan Zahavi (2010). Naturalized Phenomenology. Dans Shaun Gallagher \& Daniel Schmicking (éds.), Handbook of Phenomenology and Cognitive Science. Dordrecht : Springer Science+Business Media B.V., p. 15 ; sauf contre-indication, les traductions des travaux en anglais dans cet article sont les nôtres.

${ }^{3}$ Renaud Barbaras (2015). The Phenomenology of Life : Desire as the Being of the Subject. Dans Dan Zahavi (éd.), The Oxford Handbook of Contemporary Phenomenology. Oxford : Oxford University Press, p. 104, nous soulignons.

${ }^{4}$ Frédéric Worms (2009). La philosophie en France au XX siècle. Paris : Gallimard, p. 554.

${ }^{5} \mathrm{Ou}$, comme dit Worms, comme « un principe pour une histoire possible de la philosophie ». Ibid., p. 553.

${ }^{6}$ Ibid., p. 9.

${ }^{7}$ Idem

${ }^{8}$ Ibid., p. 14.

${ }^{9}$ Ibid., p. 562.

${ }^{10}$ Ibid., p. 562-563.
} 
«naturaliser» la phénoménologie ${ }^{1}$ et la phénoménologie de la vie (de Renaud Barbaras par exemple) entretiendraient des relations à la fois ouvertes et tendues dans la mesure où ils seraient structurés autour de problèmes philosophiques empiétant les uns sur les autres, contribuant de la sorte à esquisser le portrait de notre propre période philosophique comme un « moment du vivant»; et force est de constater que la convergence esquissée ci-dessus, montrant comment un certain nombre de projets phénoménologiques contemporains se structurent à la fois autour du problème de la fondation de la phénoménologie ainsi qu'autour du besoin d'un concept phénoménologiquement déployé de la nature, crédibilise largement une telle suggestion. Le propos de ce travail n'est, quoi qu'il en soit, certainement pas de discuter la pertinence de cette perspective historique récente sur la phénoménologie contemporaine en tant que telle ni de compléter la fresque de notre propre «moment philosophique $»^{2}$. Le propos de ce travail est bien plutôt, s'appuyant sur ce que l'esquisse wormsienne peut avoir d'éclairant, de souligner la pertinence philosophique des problèmes de la nature et de la fondation pour la phénoménologie et ses développements à venir. Or si Worms semble ici, et comme nous jusqu'à présent, inclure dans un même ensemble théorique le projet de naturalisation de la phénoménologie et les «phénoménologies de la vie», il faut cependant préciser qu'une telle inclusion ne va pas de soi. Les pensées que l'on peut appeler "phénoménologies de la vie », d'un côté, et la naturalisation de l'husserlianisme ${ }^{3}$, de l'autre, si elles constituent assurément deux terrains propices à une même réflexion sur les "pré-conditions » de la phénoménologie, n'en demeurent pas moins deux gestes qui se pensent comme essentiellement différents, voire

\footnotetext{
${ }^{1}$ Jean Petitot, Francisco Varela, Bernard Pachoud \& Jean-Michel Roy (éds.) (1999). Naturalizing Phenomenology: Issues in Contemporary Phenomenology and Cognitive Science. Stanford : Stanford University Press. Désormais abrégé NP.

${ }^{2}$ Dans une telle perspective, on devrait évidemment s'interroger sur la place qu'y occuperaient Evan Thompson et son Mind in Life de 2007.

${ }^{3}$ L'utilisation du terme "husserlianisme» pourrait paraître maladroite. Nous en faisons cependant usage pour des raisons de commodité, dans la mesure où il est également employé par les éditeurs du volume collectif de 1999 (NP). Il nous servira à désigner, dans le cadre de cet article, le Husserl pour ainsi dire canonique de la phénoménologie transcendantale publiée du vivant de Husserl lui-même et son aversion traditionnellement soulignée pour le naturalisme, tel que le présente par exemple Dan Zahavi dans ses contributions au débat sur la question. Pour une lecture divergente de Husserl, on pourra consulter avec profit la contribution de Natalie Depraz au volume collectif de 1999 intitulée «When Transcendental Genesis Encounters the Naturalization Project », NP, p. 464-489.
} 
contradictoires ${ }^{1}$. S'il nous semble philosophiquement judicieux d'insister sur leurs accointances, il est nécessaire de justifier une telle opération en mettant d'abord en évidence ce qui permet de défaire leur opposition.

Dans cette perspective, on rappellera que Barbaras annonce sa phénoménologie de la vie comme devant «mettre en question» «le partage de l'empirique et du transcendantal ${ }^{2} \gg$. Or comme l'écrivait récemment Daniel Andler dans un livre important consacré au naturalisme :

Tant qu'il n'est pas spécifié et mis en œuvre, le naturalisme n'est guère autre chose qu'une attitude, combinant la méfiance à l'égard de toute hypothèse divisant le réel en deux régions et la confiance dans la capacité humaine à le connaître en aiguisant son regard ${ }^{3}$.

Ainsi, d'une part, le naturalisme peut très bien s'accommoder du rejet du «partage de l'empirique et du transcendantal» opéré par Barbaras et il pourrait même s'en saisir comme de sa condition de possibilité : le geste de Barbaras constitue bel et bien un refus de diviser a priori la réalité en deux régimes d'existence, nous y reviendrons. D'autre part, comme l'indique encore Andler, redoublant l'accointance avec le rejet du « partage » barbarassien, « le naturalisme n'est qu'une intuition vaporeuse, un je-ne-sais-quoi, tant qu'on ne peut lui assigner une notion de nature », ainsi qu' "une stratégie pour en dessiner le périmètre et s'assurer qu'elle embrasse effectivement le tout de l'existant ${ }^{4}$. » En clair, à son niveau le plus radical, le naturalisme exhibe non seulement des similarités conceptuelles fondamentales avec la phénoménologie de la vie, mais il est aussi eidétiquement exempt de toute forme d'a priori ontologique : conceptuellement, tout ce que le naturalisme exige a priori réside dans le refus de la division de la réalité « en deux régions » ainsi que dans l'objectif que représente un concept de nature permettant de rendre raison « du tout de l'existant». Ainsi, il apparait que la possibilité d'une accointance philosophique fondamentale entre le projet de «naturalisation » de la phénoménologie d'une part, et la phénoménologie de la vie, d'autre part, se joue dans la possibilité qu'offrent ces deux perspectives - hétérogènes de prime abord — d'interroger les «pré-conditions » du

\footnotetext{
${ }^{1}$ Voir Barbaras 1999. Ce qui ne contredit en rien l'analyse de Worms dans la mesure où, comme il le souligne, les relations entre les travaux philosophiques peuvent également être oppositionnelles, op. cit., p. 14.

${ }^{2} I P V$, p. 8-9.

${ }^{3}$ Daniel Andler (2016). La silhouette de l'humain. Paris: Gallimard, p. 13, nos italiques.

${ }^{4}$ Ibid., p. 333, nos italiques.
} 
régime de pensée phénoménologique, et, plus précisément, de les interroger à partir du problème ontologique posé par la relation qu'entretiennent le naturel, ou le vital, et le phénoménal.

Articuler son rapport à l'empirie et rendre compte de ses propres « préconditions », tel est donc le nœud de contraintes que la phénoménologie a engendré dans le cours de ses développements les plus récents. Notre problème sera, face à un tel constat, de savoir à quelles conditions une articulation phénoménologique de la vie et de la nature est pensable, si la phénoménologie doit penser son propre rapport à la vie et à la nature, ou si l'une ou l'autre ne devrait finalement pas revendiquer son autonomie eu égard à la question des "pré-conditions » de la phénoménologie. Autrement dit encore, il s'agira de savoir si «la fondation authentique de la conscience» phénoménologique recherchée par Barbaras doit avoir lieu « dans la vie» ou dans la nature ; l'enjeu sera donc de savoir, enfin, ce que devient une phénoménologie qui achèverait cette démarche sur ses propres "pré-conditions » ainsi que de mettre au jour les réquisits théoriques d'un tel achèvement. Cet article entend ainsi non pas proposer un déploiement complet du concept de nature ou de vie à même de répondre aux questions tout juste soulevées, mais bien étudier les déterminations conceptuelles qu'ont reçues la vie et la nature en phénoménologie contemporaine, à la fois dans le but d'en éclaircir la signification philosophique du point de vue d'une réflexion sur les "préconditions » de la phénoménologie, ainsi que d'esquisser les développements philosophiques qui devront en découler. De tels efforts s'imposent à notre sens comme les prolégomènes nécessaires à de futures élaborations phénoménologiques de la nature ou de la vie et méritent par là même d'être étudiés afin de comprendre quel devra être le point de départ d'une conceptualisation de la nature ou de la vie permettant à la phénoménologie d'être consciente de ses propres "pré-conditions» et de pouvoir ainsi engager sa propre fondation. Dans cette perspective, nous prendrons un premier temps de clarification conceptuelle destiné à souligner les enjeux les plus fondamentaux du débat sur la naturalisation de l'husserlianisme. Dans un deuxième temps, nous examinerons, sur cette base, comment la phénoménologie contemporaine a tenté de penser le naturel du projet de naturalisation et son rapport avec la vie. Nous reviendrons, dans un troisième temps, sur la portée de telles conclusions à partir de leur articulation avec la pensée de Barbaras afin d'évaluer, enfin, à quelles conditions la phénoménologie peut s'articuler à la vie ou à la nature. 


\section{Variations husserliennes I : le projet naturaliste}

Le « projet iconoclaste ${ }^{1} »$ de Jean Petitot, Francisco Varela, Bernard Pachoud et Jean-Michel Roy (1999) a bien de quoi surprendre ${ }^{2}$ : chercher à comprendre «comment la phénoménologie husserlienne peut contribuer à la facette naturalisante de la recherche contemporaine d'une théorie scientifique de la cognition » et demander "jusqu'où une naturalisation effective de la théorie de la cognition peut, à son tour, transformer la phénoménologie husserlienne ${ }^{3} »$, ressemble au premier abord, et dans les deux cas, à une pure et simple «erreur de catégorie ${ }^{4}$ ». Il est en effet de notoriété publique que la phénoménologie husserlienne n'est pas exactement une philosophie naturaliste :

Ce qui caractérise toutes les formes du naturalisme extrême et radical [...] c'est, d'une part, qu'elles réduisent à un fait de nature la conscience [...] et, d'autre part, qu'elles réduisent à des faits de nature les idées [...]. En naturalisant les idées, cette philosophie s'annule elle-même sans s'en rendre compte 5 .

Au sein des débats contemporains, Dan Zahavi apparaît comme l'une des plus husserliennes des voix critiques - sans jamais verser dans l'hostilité à l'endroit du projet de 1999 ; il rappelait par exemple en 2010 que « selon Husserl, la limitation décisive du naturalisme réside en ce qu'il est incapable de reconnaître la dimension transcendantale de la conscience », raison pour laquelle « un phénoménologue qui embrassait le naturalisme [...] aurait cessé d'être un philosophe ${ }^{6} »$. Ce sont là les conséquences philosophiques, parmi bien d'autres, de la mise en lumière husserlienne du thème devenu canonique de «1" "ego cogito" comme subjectivité transcendantale », mise en lumière

\footnotetext{
${ }^{1}$ Wolf Feuerhahn (2011). Un tournant neurocognitiviste en phénoménologie ? Sur l'acclimatation des neurosciences dans le paysage philosophique français. Revue d'Histoire des Sciences Humaines, 2 (25), p. 63.

2 Dan Zahavi (2004). Phenomenology and the Project of Naturalization. Phenomenology and the Cognitive Sciences, 3 (4), p. 332.

${ }^{3} N P$, p. xiv.

${ }^{4}$ Dan Zahavi (2013). Naturalized Phenomenology: A Desideratum or a Category Mistake ? Royal Institute of Philosophy Supplement, 72 (supplement 1), p. 23-42.

${ }^{5}$ Edmund Husserl (2009). La philosophie comme science rigoureuse. Trad. M. de Launay. Paris : Puf, p. 20.

${ }^{6}$ Dan Zahavi, art. cit., 2010, p. 5 et 7. Pour un aperçu synthétique des critiques husserliennes du naturalisme voir p. 4-7.
} 
exposée avec force dans les Méditations cartésiennes et conduisant notamment Husserl à conclure ces mots bien connus à propos de l'être naturel :

En tant qu'être absolument antérieur, l'être du pur ego et de ses cogitationes précède en fait l'être naturel du monde [...]. Le sol ontologique naturel est secondaire quant à la validité de son être, il présuppose toujours le sol transcendantal ${ }^{1}$.

Que le naturalisme s'annule lui-même en tant que philosophie est donc corrélé, selon Husserl, à son statut de discours éternellement voué à la secondarité ontologique. Dans la mesure où ces problèmes ont été explorés par une littérature aussi rigoureuse qu'abondante ces dernières années ${ }^{2}$, nous nous en tiendrons à ce bref rappel nécessaire à l'intelligibilité de notre propos.

Dans la perspective d'une réflexion sur l'articulation phénoménologique de la vie et de la nature, il nous semble en revanche essentiel de revenir sur les motivations principielles du travail de 1999, dont l'introduction propose un aperçu aussi clair que conséquent : son point de départ est

l'idée $[\ldots]$ qu'une théorie scientifique effective de la cognition doit expliquer la phénoménalité, c'est-à-dire [...] le fait que pour tout un ensemble de systèmes cognitifs, et pour le système humain en particulier, les choses ont des apparences ${ }^{3}$.

La phénoménalité est rapidement problématisée sur le terrain de la philosophie contemporaine de l'esprit, en regard du

problème de la relation des sciences cognitives aux données phénoménologiques [...] Selon l'heureuse expression de Joseph Levine (1983), la crainte

\footnotetext{
${ }^{1}$ Edmund Husserl (2007). Méditations cartésiennes. trad. sous la direction de M. de Launay. Paris : Puf, p. 61 et 64. Il vaut la peine de rappeler ici que Natalie Depraz offre par ailleurs de bonnes raisons de revenir sur une telle lecture canonique et à bien des égards trop rapide de l'ego transcendantal husserlien dans sa propre contribution à $N P, 1999$. Notre proposition dans le cadre de ce travail prend cependant appui sur d'autres bases que les siennes, raison pour laquelle nous nous permettons de laisser de côté une exploration plus approfondie de ses travaux pour le moment.

${ }^{2}$ On pourra ainsi consulter, outre le volume de 1999, par exemple les travaux de Dan Zahavi déjà mentionnés ou de Shaun Gallagher, lesquels reviennent parfois sur les mêmes extraits de l'œuvre husserlienne que ceux que nous convoquons ici.

${ }^{3}$ Jean-Michel Roy et al., Beyond the Gap. Dans NP, p. 1.
} 
est que les sciences cognitives souffrent d'un «gouffre explicatif ». [...] La tentative de naturaliser la phénoménologie [...] pourrait [...] être vue comme une tentative de fermer ce gouffre explicatif $[\ldots]^{1}$.

Un exemple de Levine lui-même précisera ce qui est ici en question : nous savons très bien associer la douleur à un certain ensemble de processus repérables dans le système nerveux (en l'occurrence « l'activation des fibres $\mathrm{C} »)$ que l'on peut décrire avec une précision tout à fait satisfaisante d'un point de vue neurocognitif. Pour autant, et c'est ici que s'ouvre le fameux « gouffre », « ce qui est laissé inexpliqué » par ces processus nerveux est la question de savoir "pourquoi la douleur devrait être ressentie comme elle est ressentie ». Autrement dit les processus nerveux n'expliquent pas le « caractère qualitatif», "l'effet que fait» la douleur, ils n'expliquent pas « ses propriétés phénoménales ${ }^{2}$ ». C'est autrement dit bien, avec le projet de 1999, de la conscience phénoménologique dans sa dimension de vécu du point de vue de son articulation au cognitif qu'il est question.

Or cette problématisation de la conscience de l'husserlianisme dans les termes de la philosophie des sciences cognitives et de l'esprit ne va pas immédiatement de soi $^{3}$. En effet, si, pour paraphraser Aristote, la conscience se dit peut-être relativement à une unité et à une seule nature, elle se dit d'abord, et surtout, en plusieurs sens ${ }^{4}$. Comme l'a souligné Ned Block, «le concept de conscience est un concept hybride», avec cette conséquence problématique: « Des concepts très différents sont traités comme un concept unique », engendrant par là une importante "confusion » et, plus précisément, une confusion «impliquant le regroupement de deux concepts de conscience très différents » en particulier : d'une part, « la conscience phénoménale, ou $P$-consciousness », laquelle " est expérience », c'est-à-dire que ses " propriétés sont expérientielles », d'autre part, "la notion non phénoménale de conscience [...] : la conscience d'accès », dont le contenu « est représentationnel », c'est-à-dire que ses états «sont nécessairement transitifs » ou encore « toujours des états de conscience $d e^{5} »$. Block propose

${ }^{1}$ Ibid., p. 3.

2 Joseph Levine (1983). Materialism and Qualia: The Explanatory Gap. Pacific Philosophical Quartelry, 64 (4), p. 357. Cf. NP, p. 11.

${ }^{3}$ Voir Bitbol, 2006, art. cit., p. 137, et Zahavi, 2004, art. cit., p. 332 et suivantes.

${ }^{4}$ Aristote. Métaphysique, Livre $\Gamma$, 1003a 30-35 (trad. Marie-Paul Duminil \& Annick Jaulin, Flammarion). David Woodruff Smith propose une configuration du problème de la naturalisation à partir des catégories d'Aristote, voir $N P$, p. 85-87.

${ }^{5}$ Ned Block (1995). On a confusion about a function of consciousness. Behavioral and Brain Sciences, 18 (2), respectivement, p. 227-228, 230-232. Pour une mise en 
en d'autres termes une clarification des différents sens de la conscience qui en passe par une distinction entre l'être phénoménalement conscient et l'être représentation de.

Si une telle analyse a le mérite de clarifier en quoi la conscience peut se dire en plusieurs sens, reste à savoir en quoi ces différents sens peuvent se dire relativement à une seule nature. Quel est le statut exact de l'articulation de la conscience phénoménale et de la conscience d'accès (puisqu'elles « interagissent $\left.»^{1}\right)$ ? Si l'introduction du volume de 1999 ne passe pas complètement à côté d'une telle distinction conceptuelle entre les différents sens de la conscience, force est pourtant de constater que la question de leur articulation effective ne sera pas véritablement explorée dans la mesure où les éditeurs choisissent d'accorder une attention plus particulière à la question de l'explanatory gap ${ }^{2}$.

En effet, ceux-ci ne tardent pas à mettre en avant l'enjeu essentiel qui caractérise leur projet: «le cœur du problème de la naturalisation » est spécifié avant tout comme un « problème ontologique »:

selon la définition tout juste donnée, le problème de la naturalisation peut être considéré comme un cas particulier d'un processus plus général de recatégorisation des divisions ontologiques de la réalité récurrentes dans le développement des idées scientifiques ${ }^{3}$.

Il s'agit bien, en d'autres termes, de problématiser depuis la question de la naturalisation de l'husserlianisme le "hard problem de la conscience " de David Chalmers. Ce dernier écrivait en effet que «le problème réellement difficile de la conscience est le problème de l'expérience », lequel trouve sa formulation la plus claire comme suit :

pourquoi le traitement [cognitif] physique devrait-il absolument donner naissance à une riche vie intérieure ? [...] Si un problème se qualifie comme le problème de la conscience, c'est celui-là.

perspective différente de cette même distinction voir Étienne Bimbenet (2015). L'invention du réalisme. Paris : Cerf, Ch. II.

${ }^{1}$ Block, art. cit., 1995, p. 231. Voir Shaun Gallagher \& Dan Zahavi (2012). The Phenomenological Mind. London : Routledge, p. 136. Désormais abrégé TPM.

${ }^{2} N P$, p. 7-11. Comme l'indique en effet Jean-Michel Roy (2000) : « Ce texte introductif ne pose pas explicitement l'opposition entre ce qui est pour $\mathrm{x}$ selon $\mathrm{x}$ et ce qui est simplement pour $\mathrm{x} \gg$. Argument du déficit d'explication et revendication phénoménologique. Intellectica, 2 (31), p. 60 (note 35).

${ }^{3} N P$, p. 46. 
Et, précise Chalmers, il s'agit bien d'une reformulation du problème de la conscience phénoménale ${ }^{1}$.

On peut ainsi conclure que le débat consacré à la naturalisation de l'husserlianisme est largement dominé par la question de la naturalisation de la conscience " phénoménale » au détriment de la conscience « d'accès ». En effet, ce geste va être repris par la majorité des discussions qui vont émerger du projet de $1999^{2}$. Or, et c'est d'une certaine manière là tout le problème, la phénoménologie pose précisément la conscience phénoménale (au sens de Block) comme étant tout à la fois une conscience d'accès, ou la conscience d'accès comme étant tout à la fois une conscience phénoménale ${ }^{3}$.

La phénoménologie est bien " une expression de l'appréhension d'un être pour par celui-là même qui l'appréhende ${ }^{4} »$. C'est là le thème-étendard de l'intentionnalité phénoménologique comme «conscience de quelque chose » dont Sartre à proposé une formulation aussi concise que précise : « toute conscience positionnelle d'objet est en même temps conscience nonpositionnelle d'elle-même ${ }^{5} »$; dire que la conscience est «positionnelle» signifie qu'elle «se transcende pour atteindre un objet, et elle s'épuise dans cette position même: tout ce qu'il y a d'intention dans ma conscience actuelle est dirigé vers le dehors ${ }^{6} »$. Et, cependant, une telle direction vers le dehors ne saurait suffire : une explication de l'intentionnalité comme simple conscience d'accès serait « absurde » puisqu'elle « serait [...] une conscience qui s'ignorerait soi-même, une conscience inconsciente ${ }^{7} »$. L'intentionnalité phénoménologique est donc conscience de quelque chose et en même temps

${ }^{1}$ David Chalmers (2010). Facing up to the Problem of Consciousness. Dans David Chalmers, The Character of Consciousness, Oxford : Oxford University Press, p. 5.

${ }^{2}$ Zahavi, art. cit., 2013, p. 24. Voir entre autres le numéro de revue édité par Carel et Meacham en 2013 (Royal Institut for Philosophy Supplement), ou Sebastjan Vörös (2014). The Uroboros of Consciousness. Constructivist Foundations, 10 (1), p. 97. Roy donne cependant de bonnes raisons de contester l'assimilation de ce « foisonnement terminologique » au sujet du «déficit d'explication » qui cache une « situation » « passablement complexe », art. cit., p. 45.

3 «Il n'est pas possible d'expliquer l'intentionnalité de mon expérience sans expliquer également l'aspect phénoménal de l'expérience, et il est impossible d'expliquer l'aspect phénoménal de l'expérience sans en référer à son intentionnalité. » TPM, p. 136. Voir également Evan Thompson (2007). Mind in Life. Cambridge : Harvard University Press, p. 264 (désormais $M L$ ).

${ }^{4}$ Roy, art. cit., p. 62.

${ }^{5}$ Jean-Paul Sartre (2003). L'être et le néant. Paris : Gallimard, p. 19, nos italiques.

${ }^{6}$ Ibid., p. 18.

${ }^{7}$ Idem. 
« rapport immédiat et non-cognitif de soi à soi », en d'autres termes, « il y a un cogito préréflexif qui est la condition du cogito cartésien ${ }^{1} »$. Ma conscience actuelle de mon espace de travail devant moi, mon écran d'ordinateur, mon chat qui dort au milieu de mes livres entassés, est conscience de mes livres, de mon ordinateur, de mon chat, elle est bien conscience d'accès se transcendant vers eux, mais elle est bien, en quelque façon, en même temps conscience phénoménale puisque mon espace de travail fait en ce moment même partie de mon expérience, elle est bien en même temps saisie non-thétique ou " préréflexive » d'elle-même.

La phénoménologie permet en d'autres termes de penser l'articulation des différents sens selon lesquels on peut dire la conscience ${ }^{2}$, Gallagher et Zahavi ont donc raison d'insister sur la nécessité d'une approche «intégrée » :

Toute discussion de la conscience intentionnelle qui laisserait de côté la question de la conscience phénoménale (et vice versa) serait sévèrement déficiente. [...] Lorsqu'on en vient à l'intentionnalité consciente, nous avons besoin d'une approche intégrée ${ }^{3}$.

Outre les éditeurs du projet de 1999, de nos jours, c'est Zahavi ${ }^{4}$, aux côtés de Gallagher, Petit, Bimbenet, Thompson, Bitbol, ainsi que de Depraz ${ }^{5}$, qui s'est

\footnotetext{
${ }^{1}$ Ibid., p. 19.

${ }^{2}$ Ce que montre TPM. Ainsi, comme l'a par exemple souligné Michel Bitbol, « le simple fait de l'apparaître » doit être distingué « aussi bien de la conscience de soi réflexive que d'une simple fonction d'accès » Une science de la conscience équitable. Intellectica, 1 (43), p. 137. Cependant, «c'est [bien] la conscience non réflexive, écrit Sartre, qui rend la réflexion possible »Op. cit., p. 19.

${ }^{3} T P M$, p. 138.

4 Nous tenons par ailleurs à signaler que nos analyses dans cette section sont redevables de son travail ((2014). Self \& Other. Oxford : Oxford University Press) ainsi que de son livre écrit avec Shaun Gallagher (TPM), lequel propose une discussion de la "pertinence » de la phénoménologie pour la philosophie contemporaine de l'esprit et les sciences cognitives et en particulier à propos de la conscience phénoménale ; le lecteur y trouvera, entre autres, une analyse étendue et clarifiante du préréflexif en phénoménologie, notamment à partir de Sartre, Husserl et Merleau-Ponty.

${ }^{5}$ Pour Shaun Gallagher, on mentionnera parmi ses écrits : (1997). Mutual enlightenment: recent phenomenology in cognitive science. Journal of Consciousness Studies, 4 (3), p. 195-214 ; (2005). How the Body Shapes the Mind. Oxford : Oxford University Press (2015). On the Possibility of Naturalizing Phenomenology. Dans Dan Zahavi (éd.), The Oxford Handbook of Contemporary Phenomenology. Oxford :
} 
fait le phénoménologue porte-parole spécialiste de ces problèmes d'intégration des différentes dimensions de la conscience et d'articulation des théories phénoménologiques avec la philosophie analytique de l'esprit et des sciences cognitives. En effet, reprenant ces problèmes de constitution d'un «niveau préréflexif » d'analyse de la conscience en dialogue avec la philosophie de l'esprit, il débouche, suivant principalement Husserl, Sartre et MerleauPonty, sur le concept de «soi expérientiel», précisément pensé dans son articulation à l'intentionnalité positionnelle de la conscience : "l'expérience de soi est simplement une question d'être pré-réflexivement conscient de sa propre conscience, et le soi expérientiel en question est précisément défini comme la subjectivité même de l'expérience.» De plus, cette "égoïté expérientielle » caractérise « le caractère auto-présentatif de l'expérience » et «le caractère perspectif de l'expérience qui y est impliqué ». Ainsi, " une forme minimale d'égoïté est un élément intégré à la vie expérientielle ${ }^{1} »$.

Pour autant, si ce passage par la phénoménologie traditionnelle et ses actualisations contemporaines nous a fait gagner en clarté relativement à la question des différents sens selon lesquels on peut dire la conscience et sous quelles modalités doit être pensée leur articulation (dans un «soi expérientiel » préréflexif), nous semblons avoir perdu de vue la question de l'unique nature relativement à laquelle tous ces différents sens peuvent être dits. Ce

Oxford University Press, p. 70-93; (2018). Decentering the Brain: Embodied Cognition and the Critique of Neurocentrism and Narrow-Minded Philosophy of Mind. Constructivist Foundations 14 (1), p. 8-21. Pour Jean-Luc Petit, outre l'écrit déjà référencé ou sa contribution au volume de 1999, on mentionnera : Alain Berthoz \& Jean-Luc Petit (2006). Phénoménologie et physiologie de l'action. Paris : Odile Jacob. Pour Evan Thompson, on insistera sur son Mind in Life, op. cit., pour Michel Bitbol sur La conscience a-t-elle une origine?, op. cit. Pour Natalie Depraz, outre ses travaux déjà cités, on mentionnera: (2004). Le tournant pratique de la phénoménologie. Revue philosophique de la France et de l'étranger, 129 (2), p. 149165 ; (2014). Attention et vigilance. À la croisée de la phénoménologie et des sciences cognitives. Paris : Puf ; Natalie Depraz \& Thomas Desmidt (2015). Cardiophénoménologie. Les cahiers philosophiques de Strasbourg, 38, p. 47-83. Natalie Depraz, Maria Gyemant \& Thomas Desmidt (2017). A First-Person Analysis of Using Third-Person Data as a Generative Method: A Case Study of Surprise in Depression. Constructivist Foundations, 12 (2), p. 190-203. Pour Étienne Bimbenet, dont on indiquera que les travaux analysent ces problèmes d'intégration depuis la question de la constitution d'une " anthropologie philosophique », on mentionnera : (2011). Le philosophe et les neurones miroirs. Dans Après Merleau-Ponty. Études sur la fécondité d'une pensée. Paris: Vrin, p. 209-229; (2015). L'invention du réalisme. Paris : Cerf.

${ }^{1}$ Zahavi, op. cit., 2014, respectivement p. 24, 28 et 88. 
n'est pas un hasard. Zahavi est sur ce point à l'origine d'un geste à notre sens problématique: « on peut défendre la notion de conscience préréflexive de soi tout en restant tout à fait neutre vis-à-vis du problème de la naturalisation ${ }^{1}$. » Plus clairement encore, dans Self \& Other, Zahavi écrit :

Lorsque je parle $[\ldots]$ de la métaphysique du soi $[\ldots]$ je ne me préoccupe pas de la question de savoir de quelle sorte d' "étoffe » un soi pourrait ultimement être fait, et je ne suggère pas qu'une investigation phénoménologique du soi peut par elle-même résoudre cette dernière question ${ }^{2}$.

C'est autrement dit sur un refus de la dimension ontologique de l'explanatory gap que s'édifie la clarification phénoménologique des différents sens selon lesquels on peut dire la conscience ${ }^{3}$. Le problème cardinal de la philosophie de l'esprit (le hard problem) n'est donc pas — ou en tous les cas pas immédiatement - celui de la phénoménologie ${ }^{4}$. Ces affirmations confirment ainsi le diagnostic posé par Stanciu, à savoir la difficulté de la phénoménologie à penser ses propres "pré-conditions ${ }^{5}$ ». Ainsi, ce que nous gagnons avec la phénoménologie en précision descriptive et en capacité d'articulation des différents sens de la conscience, gain dont témoigne entre autres la finesse des analyses phénoménologiques portant sur le préréflexif, nous le perdons en aplomb ontologique, c'est-à-dire en capacité à répondre à la question de la fondation et de l'articulation au naturel et au vital dont nous avons fait mention ; en clair, nous le perdons en capacité à juguler le risque de « lévitation » et risquons de laisser l'analyse phénoménologique " suspendue dans le vide ».

Ainsi, le geste de Petitot, Varela, Pachoud, Roy et des contributeurs ultérieurs au débat consistant à problématiser la phénoménalité husserlienne sur le terrain de la philosophie contemporaine de l'esprit et des sciences cognitives pose à la phénoménologie une question importante puisqu'elle a trait aux "pré-conditions » même de la phénoménologie : l'horizon promis par le livre de 1999 n'est autre que celui de la réunion «naturelle» du hard

${ }^{1}$ TPM, p. 63.

${ }^{2}$ Zahavi, op. cit., 2014, p. 18.

${ }^{3}$ Il y a bien entendu des exceptions : Natalie Depraz, Francisco Varela et Pierre Vermersch (2011). À l'épreuve de l'expérience. Bucarest : ZetaBooks, et sa "portée ontologique de l'expérience subjective, à savoir le mode d'inscription du sujet dans le monde » ou son « ontologie transcendantale » p. 265.

${ }^{4}$ Ce qu'ont explicité Shaun Gallagher et Dan Zahavi, TPM, p. 27-28.

${ }^{5}$ Stanciu a également indiqué une ambivalence chez Patočka à propos de l'ontologie, art. cit., p. 310 et 314 . 
problem avec celui de la description de l'intentionnalité ${ }^{1}$. Ces clarifications notionnelles effectuées, il convient à présent d'évaluer les propositions philosophiques issues de ce débat à l'aune de la question du rapport de la phénoménologie à la vie et à la nature. Nous nous tournerons ainsi vers la tentative la plus élaborée de naturalisation de la phénoménologie pour en questionner la signification, avant d'en proposer une articulation à la "phénoménologie de la vie» de Barbaras et d'en évaluer ainsi la portée du point de vue d'une réflexion sur les «pré-conditions » de la phénoménologie.

\section{Variations husserliennes II : la nature.}

Si Zahavi refuse de mêler la phénoménologie à une quelconque ontologie de la conscience, on trouve pourtant chez lui des indications précieuses relatives à la question de la naturalisation de l'husserlianisme :

En discutant la relation entre la phénoménologie et le naturalisme, nous ne devrions pas faire l'erreur de laisser le concept de nature inexaminé. Selon un geste parallèle, on pourrait faire une remarque similaire à propos de la notion de transcendantal ${ }^{2}$.

Ce double réexamen programmatique nous exhorte en premier lieu à « repenser le concept même de nature » et cette indication pointe bien presque paradoxalement - en direction d'un travail ontologique :

Peut-être devrions-nous réaliser qu'il est nécessaire d'opérer avec une notion plus riche de nature, une qui ait de la place pour des problèmes tels que le sens, le contexte, les perspectives, les affordances et les sédiments culturels ${ }^{3}$.

Il s'agit là de la spécification la plus précise et la plus stimulante du problème : Zahavi dit ici clairement ce que bon nombre d'auteurs ont esquissé parfois trop rapidement ${ }^{4}$. À l'inverse de cette frilosité, Sebastjan Vörös et

${ }^{1}$ « l'intentionnalité a un aspect de première personne qui en fait une partie du hard problem » TPM, p. 136 ; cf. David Morris (2013). From the Nature of Meaning to a Phenomenological Refiguring of Nature. Royal Institute of Philosophy Supplement, 72 (supplement-1), note 1, p. 317-318.

${ }^{2}$ Dan Zahavi, art. cit., 2010, p. 15.

${ }^{3}$ Idem, nos italiques.

${ }^{4}$ On trouve en effet de nombreuses circonvolutions autour de l'idée formulée ici clairement par Zahavi depuis un certain nombre d'années, à commencer par l'ouvrage de 1999 lui-même. Voir Vörös, art. cit., p. 96 note 1. 
Shaun Gallagher ont pris cette injonction très au sérieux. Pour le premier, en examinant « le problème de la naturalisation de la phénoménologie [comme] complémentaire de la phénoménologisation de la nature», cette complémentarité est pensée comme un "échange dialectique », lequel doit conduire, indique Vörös - suivant ici Zahavi —, à « une reconceptualisation radicale du concept de nature ${ }^{1} \gg$. Pour autant, et malgré des développements très informés, l'auteur avoue n'avoir pas véritablement dépassé le stade de la déclaration d'intention philosophique ${ }^{2}$. Pour le second, un article très récent promet - dès son titre («Repenser la nature : la phénoménologie et une science cognitive non-réductionniste $\left.{ }^{3} »\right)$ - une reconceptualisation de ce même concept de nature depuis une perspective philosophique de la plus haute importance, celle de « la notion de réduction scientifique » dans son rapport «à une conception scientifique classique de la nature ». Dans une telle perspective, suivant Gallagher, « repenser le concept de nature lui-même» à travers la phénoménologie "sape le programme réductionniste et redéfinit ce que signifie pour la phénoménologie d'être naturalisée ${ }^{4} »$. Dans la mesure où de tels développements nous semblent absolument essentiels à la compréhension du problème que nous abordons dans le cadre de cet article, nous leur réserverons une considération plus attentive au moment d'interroger la direction que devrait prendre la question de la naturalisation de l'husserlianisme ${ }^{5}$. Il faut néanmoins déjà insister ici sur l'importance d'une telle idée d'enrichissement de la nature ou de complémentarité des gestes de naturalisation et de phénoménologisation. Elle nous semble en effet être la seule direction philosophiquement viable pour que la phénoménologie puisse penser ses propres «pré-conditions ».

Or il se trouve qu'Evan Thompson s'est approprié, en 2004, la question de la naturalisation de la phénoménologie par un geste qui non

\footnotetext{
${ }^{1}$ Vörös, art. cit., respectivement p. 96 et 101. Il vaut la peine ici de mentionner les formulations de Natalie Depraz dans sa contribution au collectif de 1999, elle mentionne en effet une «nature revisitée par la phénoménologie », c'est-à-dire une « dénaturalisation de la nature et une déspiritualisation de l'esprit ». Dans NP, p. 470 et 471 .

2 « la nature exacte de cette reconceptualisation [...] n'est [...] pas claire, mais certaines propositions faites par Varela [...], Thompson $(2007,2011)$ et Barbaras (2008, 2012, 2013) [...] semblent offrir un point de départ prometteur. »Ibid., p. 114.

3 Shaun Gallagher (sous presse). Rethinking nature: Phenomenology and a nonreductionist cognitive science. Australasian Philosophical Review.

${ }^{4}$ Idem.

${ }^{5}$ Voir infra.
} 
seulement relance la question posée par le livre de 1999 sur son terrain initial (depuis le hard problem) mais inscrit également directement cette relance dans la perspective de quelque chose comme une phénoménologisation de la nature ; il insiste en effet sur l'importance de comprendre la question comme une « refonte des termes du hard problem », refonte guidée par « l'idée d'une forte continuité de la vie et de l'esprit ${ }^{1}$. » De cette intention va naître, en 2007, son Mind in Life qui en est à bien des égards un substantiel approfondissement ${ }^{2}$. L'importance de la « refonte»y est en effet étayée ainsi :

le hard problem n'est [...] pas tant difficile qu'impossible. [...] [II] ne peut être résolu aussi longtemps que la conscience et la vie sont conceptualisées d'une manière telle qu'elles s'excluent intrinsèquement l'une l'autre ${ }^{3}$.

Thompson va ainsi déboucher sur une conclusion sur le statut de la nature qui résonne harmonieusement avec Zahavi et Vörös :

la conception physicaliste de la nature [...] ne règne plus, et à la place la nature est réexaminée depuis un angle phénoménologique. De cette manière, nous nous trouvons dans le besoin d'utiliser certains concepts, $[\ldots]$ qui sont essentiellement mixtes ou hétérogènes, au sens où ils ne peuvent être pris en compte au sein des catégories dichotomiques du physique et du phénoménal, ou de l'objectif et du subjectif ${ }^{4}$.

Autrement dit, la phénoménologisation de la nature est ici pensée à partir d'un double rejet : celui du physicalisme naturel ainsi que des dichotomies qui lui sont conceptuellement associées. Cette phénoménologisation uniquement négative (double rejet) pourrait cependant être le motif d'une inquiétude et celle-ci trouve de quoi se légitimer dans la démarche de Thompson : celui-ci ne prend en effet jamais la peine d'éclairer véritablement le sens de cette hétérogénéité constitutive de la nature. Elle ressemble de ce fait beaucoup trop pour qu'on puisse la prendre au sérieux à ce que Merleau-Ponty appelait « mauvaise ambiguïté », c'est-à-dire à une ambiguïté qui ne clarifie en rien le statut véritable de l'objet qu'elle circonscrit (ici, la

\footnotetext{
${ }^{1}$ Evan Thompson (2004). Life and mind : From autopoiesis to neurophenomenology. Phenomenology and the Cognitive Sciences, 3 (4), 2004, p. 385.

${ }^{2} M L$; on pourra consulter Zahavi, art. cit., 2013, p. 39-40 pour une synthèse efficace du geste de Thompson.

${ }^{3} M L$, p. 225.

${ }^{4}$ Ibid., p. 359.
} 
nature hétérogène) ${ }^{1}$. Thompson nous laisse autrement dit en plein malaise explicatif qu'il «se borne à subir » et qui, par là même, «s'appelle équivoque $^{2} \gg$. Alva Noë a récemment pointé le nœud de cette équivoque dans la tentative de Thompson. En effet, soulignant, avec Thompson, que «la conscience et la cognition sont eux-mêmes des aspects du développement de la vie», Noë demande si cela ne revient pas, à la fin, à simplement «substitu[er] un gouffre explicatif par un autre », c'est-à-dire à substituer le problème de l'explanatory gap par celui « des origines de la vie ${ }^{3} »$.

En d'autres termes, bien qu'il puisse être lu comme une authentique tentative d'assimilation phénoménologique de la nature, Mind in Life semble en définitive largement insuffisant pour enrichir la prise en charge philosophique de la nature ou pour "phénoménologiser » la nature. Il nous semble essentiel de ce fait de nous tourner vers le travail de Barbaras et en particulier vers son Introduction à une phénoménologie de la vie, dans laquelle il rencontre un système de problèmes qui concernent directement les questions abordées ici, tout en proposant une prise en charge de ceux-ci tout à fait soucieuse de ne pas rester dans l'équivoque.

\section{Variations husserliennes III : la vie}

Ce n'est pas par hasard si nous le retrouvons à ce stade de notre investigation : au pays des phénoménologues en lévitation, Barbaras est une exception

\footnotetext{
${ }^{1}$ Maurice Merleau-Ponty (1962). Un inédit de Maurice Merleau-Ponty. Revue de Métaphysique et de Morale. Année 67 (4), p. 409. Sur la «mauvaise ambiguïté » merleau-pontienne, voir Renaud Barbaras (1990). De l'être du phénomène. Grenoble : Jérôme Millon, qui la décrit comme un geste consistant à « demeurer au plan descriptif, se contenter de mettre à jour un domaine qui reste à penser. » p. 36. C'est en ce sens qu'il désigne les œuvres du premier Merleau-Ponty « comme des œuvres archéologiques » (p. 59). Voir également Étienne Bimbenet (2004). Nature et humanité. Paris : Vrin, p. 31. Sur les problèmes posés par le raisonnement seulement négatif voir Renaud Barbaras (2001). Merleau-Ponty et la psychologie de la forme. Les Études Philosophiques, 2 (57), p. 151-163.

${ }^{2}$ Maurice Merleau-Ponty (1989). Eloge de la philosophie. Paris : Gallimard.

${ }^{3}$ Alva Noë (2004). Action in Perception. Cambridge-London: MIT Press, p. 230231, p. 249 pour la note qui signale la dette à l'égard de Thompson. Noë indique qu'il a eu accès à un livre "Radical Embodiment (à paraître)» de Thompson qui développait «l'hypothèse de la continuité esprit-vie» p. 249. Nous faisons ici l'hypothèse qu'il s'agissait d'une version antérieure de Mind in Life.
} 
d'un aplomb des plus newtonien ${ }^{1}$. En effet, refusant d'abord catégoriquement la non-prise en charge de l'ontologie par la phénoménologie, à travers la question du « sens d'être » de la subjectivité phénoménologique dans l'œuvre de Merleau-Ponty, il dénonçait sa traditionnelle «insularité » solipsisante ${ }^{2}$.

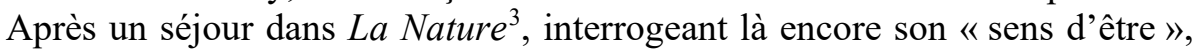
pour réaffirmer que « la philosophie de la nature qui affleure chez le denier Merleau-Ponty » pourrait bien être le «signe» du "mode d'accomplissement le plus exigeant» de l'investigation phénoménologique et non de son « abandon ${ }^{4} »$, son parcours va donner lieu, en 2008, à un retour

au projet constitutif de la phénoménologie pour tenter de comprendre en quoi elle est confrontée sans cesse à une question qu'elle ne peut pourtant prendre conceptuellement en charge.

Ce qui est ici en question n'est autre que « la difficulté de la phénoménologie à regarder en face et à définir en propre cette vie dont elle ne cesse pourtant de parler». Un tel projet revient à « interroger le sens d'être de la vie pour lui-même », ce qui implique notamment de demander « quel est le sens d'être de la vie en tant qu'elle est susceptible de se faire conscience. »

Barbaras nous propose bien, en d'autres termes, une immense plongée au plus profond du «sens originaire du vivre», lequel «n'est autre que l'exister du sujet. » À l'issue d'une traversée des grandes pensées qui abordent ce «problème de la vie» Barbaras va chercher à « dépasser le dualisme ontologique ${ }^{5} »$. Ce dépassement s'éclaire à la lumière d'une idée jonassienne qu'il prend très au sérieux, celle d' $\mathrm{d}^{\text {' }}$ ontologie de la mort ${ }^{6}$ » qu'il s'agit pour lui de « suspendre ${ }^{7} »$, en un sens fort :

\footnotetext{
${ }^{1}$ Il ne s'agit pas ici d'instruire un procès mais d'insister sur une tendance qui nous semble problématique.

${ }^{2}$ Renaud Barbaras, De l'être du phénomène, op. cit.; (1998). Le tournant de l'expérience. Paris : Vrin, 1998.

${ }^{3}$ Maurice Merleau-Ponty (1995). La Nature, Cours du Collège de France, notes. Paris : Seuil.

${ }^{4}$ Renaud Barbaras (2000). Merleau-Ponty et la Nature. Chiasmi International, 2, p. 60.

${ }^{5} I P V$, p. $11,20,62,128,266$ et 311.

${ }^{6}$ Ibid., p. 134, c'est-à-dire une ontologie " où la vie elle-même est ressaisie sous l'horizon de la mort» ainsi «la vie n'est pas comprise selon son sens d'être véritable ». Idem.

${ }^{7}$ Ibid., p. 258.
} 
on n'est en mesure d'accéder au sens d'être de la vie qu'à la condition d'effectuer une époché de la mort, sous la double forme d'une suspension de la mort à laquelle la vie est exposée et de l'ontologie naturaliste dont procède cette définition de la vie ${ }^{1}$.

En effet, selon lui, cette « ontologie de la mort [...] n'est [...] que l'autre nom de l'ontologie réaliste ou naturaliste ${ }^{2} »$.

Le dépassement du dualisme est donc assuré par un point de départ dans le vital lui-même qui installe la méditation spéculative hors du « vocabulaire de la dualité ${ }^{3} »$. Pour autant, précise Barbaras, ce dépassement «ne peut être au profit d'un [simple] [...] monisme ontologique » mais bien d' "un monisme que nous pourrions qualifier de phénoménologique», en tant qu'il «met en avant l'irréductibilité du champ phénoménal et tente de penser ses conditions à partir de ce champ lui-même ${ }^{4} »$. Quoi de plus logique, si le concept de vie qui autorise ce dépassement a été lui-même conçu précisément comme une alternative aux ontologies réalistes et naturalistes ? Il s'agit bien, en effet, d'éviter à tout prix «la réification», «tentation presque inévitable de la pensée ${ }^{5}$.» Ici, en fin de compte, conscience phénoménale et conscience d'accès trouvent leur unité dans la vie.

Ce monisme phénoménologique pose cependant un problème massif que Bitbol a sobrement et néanmoins lucidement relevé. En effet, au cours de son investigation attentive de "l'expérience », ou « singularité organique de

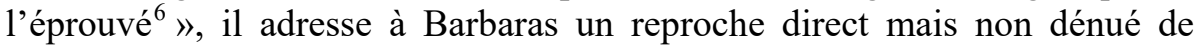
pertinence :

Barbaras a lui aussi fini par choisir un nom de substitution : la vie, reconduite en-deçà du clivage entre vécu et corps vivant [...]. Mais la vie ne fait pas exception à la règle d'airain de la détermination; comme toute chose désignée, elle est différenciée-de ; elle se différencie pour sa part du nonvivant, du cadavre, de l'inerte, de l'objet des sciences physico-chimiques?

\footnotetext{
${ }^{1}$ Ibid., p. 230.

${ }^{2}$ Idem.

${ }^{3}$ Ibid., p. 265.

${ }^{4} \mathrm{Ibid} .$, p. 311.

${ }^{5}$ Ibid., p. 313. Voir également, (2012). Sauver d'une réification de la conscience la tâche de la phénoménologie. Les Études philosophiques, 1 (100), p. 49-63.

${ }^{6}$ Michel Bitbol (2014). La conscience a-t-elle une origine? Paris : Flammarion, p. 295.

${ }^{7}$ Ibid., p. 298.
} 
Nous laisserons ici ouverte la question de savoir si le concept barbarassien de vie peut être si abruptement déclaré coupable de détermination (synonyme, chez Bitbol, de «réification ») : le soin porté par Barbaras à la vie dans le but d'en conceptualiser l'intrinsèque non-objectalité nécessiterait qu'une telle accusation soit beaucoup plus étayée qu'elle ne l'est ici. En revanche, la conséquence de cette accusation est plus préoccupante : Bitbol souligne à juste titre que la vie ainsi conceptualisée semble rendre impossible la prise en charge philosophique du «non-vivant ». C'est autrement dit bien l'ensemble de la nature non-vivante qui semble avoir été évacuée hors du concept par «l'époché de la mort » barbarassienne et qui n'est, en l'état, pas réintégrable à celui-ci : la suspension de l'ontologie naturaliste réalisée par Barbaras semble bien l'empêcher d'atteindre conceptuellement les objets inertes qui peuplent notre univers; une fois passée la lisière du vital, comment rendre raison, dans un tel régime théorique, de phénomènes aussi courants que l'ébullition de l'eau ou aussi spectaculaires que les éruptions volcaniques ? Il est donc nécessaire de reposer ici le problème que Stanciu découvrait avec Patočka, à savoir, celui qui consiste à demander comment inscrire le phénoménal même au sein d'une physis. En effet, si Barbaras semble bien avoir commencé exactement là où Thompson s'était arrêté, il n'est pour le moment pas certain que nous ayons significativement progressé quant au problème de substitution des explanatory gaps que pointait Noë, dans la mesure où le problème de la possibilité philosophique d'une non-vie pour une phénoménologie vitale semble finalement n'être qu'une autre manière de pointer le problème de l'origine de la vie.

Dans un texte de 2015 intitulé «La phénoménologie de la vie», on trouve cependant de quoi nuancer cette lecture. Il s'agit cette fois pour Barbaras de reprendre la question initiale de la vie à partir de la «profonde parenté ontologique entre le sujet et le monde», laquelle est considérée « comme le véritable fondement de la relation entre sujet et monde»:

au-delà du sujet et du monde, au-delà de la vie du sujet et des mouvements qui prennent place au sein du monde, il y a un archi-mouvement des processus « naturels » (au sens de $\varphi v ́ \sigma ı \varsigma)[\ldots]$.

Autrement dit, dans un geste qu'il situe à la suite de Patočka, Barbaras

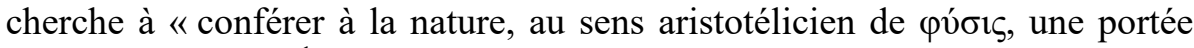
phénoménologique ${ }^{1}$. » Seulement, bien loin de maintenir un tel cap qui pourrait nous conduire vers le concept adéquat de nature phénoménologisée,

${ }^{1}$ Barbaras. The Phenomenology of Life. Dans op. cit., p. 102-104.

21 
c'est encore la vie qui va devoir se porter garante de la nature : « Il y a une vie du monde dans le sens qui est évoqué par la compréhension aristotélicienne de la $\varphi v ́ \sigma ı \varsigma$, une vie des choses. »

Même si Barbaras se défend de toute naïveté vitaliste, force est de constater que cette vie - désormais pensée comme « archi-vie» - repose exactement le problème soulevé par Bitbol à propos du «non-vivant», $a$ fortiori lorsque Barbaras écrit : "Comme Canguilhem l'a souligné, [...] la vie ne peut être un empire dans un empire ; la vie est le seul empire. » Plus clairement encore : «En vérité, "Vie" est le nom donné au sens de l'Être : rien qui prétend être ne peut se tenir hors de son étreinte ${ }^{1}$. » Il est ici difficile de résister à la tentation d'objecter à une archè par une autre et de remarquer qu'à l'image du «corrélationisme» dénoncé par Quentin Meillassoux ${ }^{2}$, la pensée de «l'archi-vie » semble incapable de faire droit à ce qu'il nomme «archifossile », c'est-à-dire "les matériaux indiquant l'existence d'une réalité ou d'un événement ancestral, antérieur à la vie terrestre ${ }^{3}$. » Doit-on en effet comprendre, chez Barbaras, et selon un exemple cher à Meillassoux, que la formation de notre globe terrestre est l'œuvre de «l'archi-vie»? Si rien ne doit échapper à «l'empire » de la vie, la réponse semble aussi claire qu'elle est difficile à accepter.

Ainsi, vouloir procéder à l'enrichissement zahavien de la nature ou à sa phénoménologisation en ne passant que par la vie s'avère au fond inefficace parce qu'en définitive «la vie ne se quitte pas; elle ne peut rencontrer le réel qu'en le projetant depuis ses propres ressources ${ }^{4} »$, une thèse déployée par Étienne Bimbenet dans L'invention du réalisme dans toutes ses implications anthropologiques et qui trouve ici l'occasion d'une application épistémologique. En d'autres termes, la phénoménologie de la vie ne nous donne qu'une nature dépossédée de toute « ancestralité ${ }^{5}$ » et de toute non-vie (l'ébullition de l'eau, l'éruption volcanique) et elle semble incapable de s'y réarticuler après la suspension délibérée de «l'ontologie de la mort ». Ainsi, si la Vie des phénoménologues contemporains permet de comprendre pour quelles raisons et dans quelle direction il est nécessaire de "phénoménologiser » la nature, il semble qu'un tel projet soit voué à buter éternellement sur les mêmes limites philosophiques telles que les révèlent les

${ }^{1}$ Ibid., p. 106, 107 et 110.

${ }^{2}$ Quentin Meillassoux (2006). Après la finitude. Paris : Seuil, p. 168.

${ }^{3}$ Ibid., p. 26.

${ }^{4}$ Bimbenet, op. cit., 2015, p. 120-121.

${ }^{5} \mathrm{C}$ 'est-à-dire de « toute réalité $[. .$.$] antérieure à toute forme recensée de vie sur la$ Terre ». Meillassoux, op. cit., p. 25-26. 
remarques d'Alva Noë et Étienne Bimbenet. On le constate chez Renaud Barbaras : la vie comme «seul empire» semble bien rendre obsolète la question des « origines de la vie » posée par Alva Noë, et pourtant, une telle question refait surface dès le moment où se pose le problème de l'« ancestralité ». Pour le dire autrement, il nous semble, à la lumière de ces analyses, que « naturaliser la phénoménologie » ou "phénoménologiser la nature », ne pourra aboutir qu'à la condition que la vie soit architectoniquement secondaire par rapport à la nature, ce qui revient à dire que la «fondation authentique de la conscience » recherchée par Barbaras devra s'opérer, non pas «dans la vie », mais bien dans une nature dont la définition et l'enrichissement paraissent à ce titre de plus en plus urgents. Pourquoi, dans ce cas, s'astreindre à une telle exploration du geste barbarassien ? Bien loin de considérer que nous aurions pu nous en passer, la pensée de Barbaras permet à notre sens de tracer le chemin d'un enrichissement adéquat de la nature, pour peu qu'on accepte de l'articuler au projet naturaliste de 1999.

\section{Ruyer et le projet naturaliste}

Que peut, malgré tout, nous apporter la vie barbarassienne du point de vue de la question qui est la nôtre ? Si la suggestion peut paraître surprenante, il nous semble pourtant qu'une réponse constructive à cette question doit en passer par une mise en perspective de son effort avec le projet de 1999. D'une certaine manière, une telle perspective de dialogue était laissée ouverte par Barbaras lui-même puisqu'il conclut sa contribution au volume de 1999 en indiquant que « la naturalisation de l'intentionnalité fait sens à la condition que la nature soit "désobjectivée" »".

Qu'est-ce que cette nature non-objectale que pointe ici Barbaras? Lisons-le encore : «L'essence de l'intentionnalité implique son appartenance à une Nature », cela ne revient pourtant pas à « une réhabilitation de quelle que forme de naturalisme que ce soit». Plus encore, «la Nature au sein de laquelle l'intentionnalité prend place est la négation même de toutes les dimensions de l'idée classique de Nature », c'est même " une anti-Nature ${ }^{2}$ ». La mise en perspective ici proposée n'était donc pas donnée d'avance, le refus du naturalisme étant on ne peut plus clair. Pour autant, une telle opposition nous semble pouvoir être défaite assez rapidement. D'abord, en

${ }^{1}$ Barbaras. The Movement of Living as the Originary Foundation of Perceptual Intentionality. Dans $N P$, p. 538.

${ }^{2}$ Ibid., p. 537. 
raison du geste que ces deux projets partagent de mise en cause des sécessions fondamentales de la réalité que nous avons souligné. Ensuite, parce que le geste de Barbaras n'est pas sans rappeler l'exigence d'une conception «plus riche» de la nature réclamée par Zahavi. Enfin, parce qu'on rappellera que le naturalisme n'implique pas nécessairement la conception classique de la nature rejetée par Barbaras ${ }^{1}$, ce dont la définition d'Andler témoigne nettement. En clair, non seulement la pensée de Barbaras n'est, en son point de départ, pas incompatible avec le naturalisme, même si elle s'y oppose dans ses conclusions, mais encore, elle semble nous promettre exactement la possibilité de penser cette "notion plus riche de nature " qui nous fait encore défaut: "désobjectivée », "effective et dynamique négation de toute positivité ou entité », "potentielle» comme « ce qui implique toute chose et donne naissance à toute chose ${ }^{2}$ », la nature de Barbaras tend bien vers un concept à même de faire droit aux réquisits annoncés comme essentiels par Zahavi en vue d'un enrichissement naturaliste de la nature ou sa phénoménologisation.

On objectera que cette nature était déjà, en 1999 , pensée à partir de la vie $^{3}$, peut-être; rien ne nous empêche pourtant de quitter à présent cette dernière pour tenter de penser la nature directement et depuis la nonobjectalité ${ }^{4}$ et sans devoir en passer par la vie. Il nous semble que Raymond Ruyer devra nous permettre d'explorer avec profit une telle perspective. Celui-ci a en effet constitué une philosophie qui répond exactement à l'exigence zahavienne d'enrichissement de la nature. Selon Ruyer, centralement, « tout existant actuel, par définition, actualise, c'est-à-dire travaille ${ }^{5}$ »

\footnotetext{
${ }^{1}$ Zahavi propose une remarque similaire dans art. cit., 2010, p. 15, ajoutant que le naturalisme a bien tendance à «monopoliser» la nature en un tel sens classique. Voir également art. cit., 2013, p. 33. De même Thierry Hoquet a récemment proposé en ce sens de «travailler le naturalisme de l'intérieur» dans (2015). L'alternaturalisme. Comment travailler le naturalisme de l'intérieur. Esprit, janv. (1), p. 48.

${ }^{2}$ Barbaras, The Movement of the Living. Dans NP, p. 538.

${ }^{3}$ Idem.

${ }^{4}$ Nous nous dirigeons ainsi dans la direction que Steven Crowell a désignée comme celle d'un «naturalisme transcendantal», lequel est structuré par l'exigence de « développer un concept transcendentalement viable de nature ». Si ces désignations nous semblent verbalement adéquates, nous n'endosserons pas le contenu pratiquement constructiviste que Crowell leur assigne. Steven Crowell (2015). Transcendental Phenomenology and the Seduction of Naturalism. Dans Dan Zahavi (éd.), op. cit., p. 41 et 43.

${ }^{5}$ Raymond Ruyer (2012). Néo-finalisme. Paris : Puf, p. 12.
} 
et un travail s'effectue toujours « selon une norme ${ }^{1} »$. Or une telle caractérisation ne s'applique pas en premier lieu à la vie ou à la conscience phénoménale, mais bien à la nature ou aux entités naturelles :

Un atome n'est pas une mécanique toute montée, et qui fonctionne. Il est activité incessante; il "se forme" sans arrêt. Or, une activité, ou une formation active, est indissociable d'une norme ${ }^{2}$

et, faut-il crucialement ajouter, d'une conscience. Une précision est impérative ici :

ces « consciences primaires » qu'envisage Ruyer ne se confondent pas avec la conscience réflexive de l'homme, [...] elles sont donc, quoique parfaitement déterminées, aveugles, et naturellement privées des fonctions supérieures qui caractérisent l'homme ${ }^{3}$.

En d'autres termes, c'est bien d'une pensée s'exposant, ainsi que le regrette Barbaras et que nous estimons au contraire nécessaire, comme «un dépassement de la vie proprement dite au profit d'un mode d'être ultime qui est celui des êtres primaires, auto-unifiées (atome, molécule, cellule, tissu, organe, organisme, etc.), dont le vivant n'est qu'un exemple ${ }^{4} »$ qu'il est ici question, c'est-à-dire finalement d'un panpsychisme en un sens bien particulier ${ }^{5}$, puisque chez Ruyer, il est structuré par une équivalence philosophique bien précise :

Toute définition de l'existence et de la liberté qui ne postule pas implicitement ce rapport : liberté $=$ existence $=$ travail, est creuse. Supprimer un de ces trois termes, c'est supprimer les deux autres. Un être n'est authentique $[\ldots]$ que dans la mesure où il fait un effort laborieux ${ }^{6}$.

Or en une vingtaine d'années de discussions sur la naturalisation de la phénoménologie, le panpsychisme n'aura été nommé explicitement qu'une

${ }^{1}$ Ibid., p. 19.

${ }^{2}$ Ibid., p. 173.

${ }^{3}$ Fabrice Colonna (2007). Ruyer. Paris : Les Belles Lettres, p. 249.

${ }^{4} I P V$, p. 180. Ce qui conduit Barbaras à abandonner précisément ce chemin esquissé par Ruyer pour des raisons exposées à la suite de ces quelques lignes.

${ }^{5}$ On pourra lire les éclairantes analyses de Fabrice Colonna dans son Ruyer pour plus de précisions sur ces questions à peine introduites ici.

${ }^{6}$ Ruyer, op. cit (2012), p. 12. Voir Colonna (2007), op. cit., p. 170-171 sur «l'équivalence $»$. 
seule fois - et trop rapidement ${ }^{1}$. C'est d'autant plus préjudiciable que la pensée ruyérienne se présente exactement comme une solution aux problèmes qui nous occupent :

Cette solution peut être énoncée en peu de mots : le problème posé par la dualité de la conscience et du corps [...], est un problème apparent, pour l'excellente raison qu'il n'y a pas de corps [au sens classique]. Le " corps » résulte, comme sous-produit, de la perception d'un être par un autre être. L'être perçu est perçu par définition comme objet [...]. Il apparaît, d'autre part, comme indépendant de l'observateur, ce qui conduit à le substantialiser ${ }^{2}$.

En d'autres termes, point d'explanatory gap à l'horizon, et cette pensée s'impose ainsi à notre sens comme un passage obligé pour les questions qui nous concernent : d'une part, elle semble bien nous offrir la possibilité de penser l'enrichissement d'une nature non-objectale comme nature «d'activité incessante ", d'autre part, elle est exactement, si l'on s'en tient à la définition d'Andler, une pensée naturaliste ; «il est [même] possible de désigner la pensée de Ruyer comme un matérialisme d'après la révolution quantique » bien que « Ruyer récuse explicitement cette appellation en raison des équivoques réductionnistes qu'elle charrie encore ${ }^{3}$. » Par ailleurs, plus significativement encore, Ruyer offre exactement la possibilité de penser ensemble, en l'occurrence au sein d'une nature non-objectale, une description cognitive et une fixation du phénoménal, dans la mesure où la considération ruyérienne du système nerveux le conduit à l'envisager à la fois, bien évidemment, comme le lieu propre du cognitif, mais également, tout à la fois comme le lieu même de la conscience phénoménale: en effet, tout comme l'atome, le système nerveux est le lieu d'une «activité incessante» de formation, c'est-à-dire, finalement, d'une activité subjective ; plus encore :

Nous n'avons pas un troisième œil pour voir notre aire visuelle occipitale, ni de troisième oreille pour entendre notre zone auditive temporale. Il faut bien que, finalement, la conscience soit unie d'une façon immédiate au cerveau en tant que tissu vivant, pour que la conscience sensorielle paraisse être une

\footnotetext{
${ }^{1}$ Morris, art. cit., p. 322. Son effort entre directement en résonnance avec le nôtre : « le hard problem n'est pas de comprendre l'esprit, mais de reconfigurer la nature " (p. 319), reconfiguration opérée à l'aide d'une « étude de l'embryogenèse » (p. 321), très ruyérienne en son principe. Cependant, c'est chez lui la vie qui vient « reconfigurer la nature » et ce problème nous incite à reléguer sa stimulante tentative à cette note.

${ }^{2}$ Ruyer, op. cit., p. 92 . Voir $I P V$, p. 159 et suivantes.

${ }^{3}$ Colonna, op. cit., p. 251.
} 
propriété du cerveau en tant qu'organe disposé macroscopiquement pour la réception sensorielle ${ }^{1}$.

En un sens, le naturel se trouve ici philosophiquement enrichi par voie de phénoménologisation, puisque le système nerveux central est toujours-déjà conscientiel; autrement dit, le cognitif et ses processus que décrivent les recherches naturalistes existe de facto toujours-déjà comme conscience phénoménale, c'est-à-dire que ce phénoménal lui est non seulement intrinsèque, mais également essentiel d'un point de vue fonctionnel. En d'autres termes, ceux de Barbaras, «l'être du corps vivant impose d'y reconnaître l'œuvre d'une conscience ${ }^{2} \gg$. Dans le cas contraire, indique en effet crucialement Ruyer, l'analyse du système nerveux serait confrontée à une régression à l'infini :

Si le mode de causalité dans les domaines cérébraux était semblable à celui des cheminements ou des propagations dans les réseaux mécaniques, dans les systèmes artificiels de circuits électriques, si le cerveau était une machine à calculer électrique, la conscience serait inutile - mais aussi, il n'y aurait pas d'arrêt dans la régression à l'infini ${ }^{3}$.

Classiquement, cela signifie qu'il n'y aurait pas, pour reprendre l'exemple ruyérien ci-dessus, d'arrêt dans la série des questions consistant à demander : comment les processus s'actualisant dans notre zone auditive donnent-ils lieu à l'audition phénoménale elle-même (le fait même d'entendre tel ou tel son)? Si l'on répond au moyen d'une entité tierce (Ruyer parle d'une « représentation du cerveau dans le cerveau »), alors la question se repose de savoir comment cette entité tierce peut donner lieu à l'audition phénoménale à partir des processus neuraux, et ainsi de suite. $\mathrm{Si}$, au contraire, on décide de

${ }^{1}$ Ruyer, op. cit., p. 45. C'est-à-dire, comme l'a souligné André Conrad, que « la subjectivité doit [...] être identifié à l'aire cérébrale ». (2014) Repenser la finalité. Critique, LXX (804), p. 397. Voir également Jean-Pierre Louis (2017). L'intériorité n'est-elle qu'un mythe ? Philosophia Scientice, 21 (2), p. 123-138 sur le «fonctionnel » : «L'état phénoménal [...] joue bien un rôle causal». En effet, « l'état mental et l'état cérébral ne font qu'un », le premier est « une propriété intrinsèque » du second, ce qui conduit Louis, de manière intéressante, à montrer qu'il n'est pas nécessaire de « concéder à Wittgenstein que l'abîme entre le cerveau et la pensée ne résulte que d'une erreur grammaticale, ni que l'intériorité ne soit qu'un mythe dont il faudrait nous débarrasser par une analyse du langage. » P. 133, 135 et 137.

${ }^{2} I P V$, p. 161.

${ }^{3}$ Raymond Ruyer (1964). L'animal, l'homme, la fonction symbolique, Paris : Puf, p. 80 . 
s'en tenir strictement aux processus observés dans notre zone auditive, alors on s'interdit purement et simplement de rendre raison de l'audition phénoménale elle-même, ce qui est absurde (puisque nous entendons effectivement des sons toute notre vie durant grâce à cette même zone auditive). Pour parler dans un langage plus contemporain, l'enrichissement du cognitif naturel que nous permet de penser Ruyer est non seulement philosophiquement heuristique mais également logiquement asséné par une démonstration par l'absurde : nier cet enrichissement phénoménal du cognitif ou le déléguer à une entité tierce conduit en effet soit à une aberration, soit à une régression à l'infini. Comme l'a sobrement indiqué Barbaras à propos de ce même mouvement de pensée ruyérien, «la subjectivité est une propriété du champ [auditif] lui-même : elle ne renvoie pas à une entité à laquelle le champ phénoménal serait relatif mais à sa phénoménalité même ${ }^{1} »$. Ainsi, avec Ruyer, pour reprendre les termes de Stanciu par lesquels nous avons ouvert le présent travail, c'est en un sens la perspective d'une physis au sein de laquelle le champ phénoménal n'est pas seulement inscrit qui est rendue pensable, mais bien une physis dont la phénoménalité constitue à la fois la densité même (la zone auditive comme lieu de l'audition phénoménale) et le principe de fonctionnement (la zone auditive comme lieu du cognitif de l'audition) $)^{2}$. Cette physis s'annonce de ce point de vue bien comme "désobjectivée », niant "toutes les dimensions classiques de l'idée de Nature » et, d'une certaine manière, également comme « une anti-Nature ». Or il faut souligner, avec Andler, qu'une telle physis prétend bien embrasser « effectivement le tout de l'existant» et refuser de diviser «le réel en deux régions $^{3}$ », c'est-à-dire qu'elle permet, selon les termes de Thierry Hoquet,

\footnotetext{
${ }^{1} I P V$, p. 161. On pourra par ailleurs consulter l'ensemble du chapitre (p. 157-181) consacré à Ruyer revenant très clairement sur la question de la régression à l'infini et lui conférant une importance capitale dans le dispositif ruyérien. En effet, selon Barbaras cette mise au jour, montrant que «le champ visuel [ou auditif] a ceci de propre qu'il est sa propre conscience ", conduit Ruyer à penser une "solidarité essentielle de la conscience et de l'étendue », que Barbaras qualifie comme étant la «découverte centrale de Ruyer» p. 162-163. Sur la régression à l'infini comme « analyse de type logique » voir Colonna (2007), op. cit., p. 40-41.

${ }^{2}$ C'est d'ailleurs ce que Barbaras a accompli (art. cit., 2015, p. 104) : «Parler de $\varphi v ́ \sigma \iota \varsigma[\ldots]$ ce n'est pas réduire la phénoménologie à une philosophie de la nature. Au contraire, c'est conférer à la nature, au sens aristotélicien de $\varphi v ́ \sigma \iota \varsigma$, une portée phénoménologique.» Nous pourrions nous en satisfaire au-delà de la simple formulation si la physis n'était ici pas secondaire par rapport à la Vie.

${ }^{3}$ Voir supra.
} 
de « travailler le naturalisme de l'intérieur » pour le « repenser $^{1} »$ à partir du non-objectal et de « l'activité incessante » décrite par le ruyérisme ${ }^{2}$.

Par ailleurs, la phénoménologie contemporaine commence à prendre conscience des possibilités offertes par une telle entreprise : Gallagher vient en effet tout juste de proposer «de repenser le concept de nature» une proposition qui, inévitablement, "redéfinit ce que signifie pour la phénoménologie d'être naturalisée ${ }^{3}$. " Un tel geste conduit Gallagher, avec MerleauPonty et la physique contemporaine à travers Niels Bohr principalement, vers une " nature relationnelle ", "dynamique », une " conception non classique de la nature ", c'est-à-dire un rejet de la "nature comprise partes extra partes $^{4}$ » ainsi que du « réductionnisme». Dans un lexique et un projet qui ne sont pas sans résonnance avec les conclusions que nous avons atteintes dans le cadre de ce travail, Gallagher précise que

Cette nature relationnelle, irréductible au cerveau et à l'objet, est la nature que la science doit expliquer. Ce concept de nature va de pair avec l'idée que les phénomènes à expliquer sont irréductibles. Repenser en ce sens la nature et le réductionnisme veut également dire que nous avons à repenser la science pas seulement la science telle qu'elle est pratiquée par le scientifique expérimentaliste, mais notre conception théorique de la science, ou la science telle que nous la connaissons. ${ }^{5}$

\footnotetext{
${ }^{1}$ Thierry Hoquet (2015), art. cit., respectivement p. 41, 42. Il nous semble cependant important de remarquer que si, verbalement, la nécessiter de repenser le naturalisme propre à Hoquet nous semble essentielle, la direction prise par ce dernier nous semble, en revanche, moins évidente à suivre. En effet, lorsqu'il écrit que «l'alternaturalisme est toujours problématique: c'est un naturalisme sceptique, accueillant la diversité et susceptible d'être autrement qu'il n'est», ou encore, lors qu'il indique que «d'un point de vue théorique, l'alternaturalisme tente un antiessentialisme qui ne soit pas un anti-réalisme » (p. 51), nous craignons, en définitive, de ne pas pouvoir identifier de quoi il est véritablement question et en particulier de ne pas comprendre quel pourrait bien être le concept de (alter)nature propre à «l'alternaturalisme».

${ }^{2}$ Anne Sauvagnargues a développé cette idée dans un article actuellement soumis pour publication intitulé «Le dynamisme organisateur et son œuf. Ruyer, et sa théorie molaire des multiplicités ».

${ }^{3}$ Gallagher (sous presse), art. cit. Texte aimablement fourni par Sh. Gallagher luimême, que nous remercions.

${ }^{4} \mathrm{Ibid}$., p. 7, 6, 3 et 5, nos italiques (excepté pour la locution latine).

${ }^{5}$ Ibid., p. 7. Voir également ses remarques dans Shaun Gallagher (2018). Dynamics and Dialectics. Postscript. Constructivist Foundations, 14 (1), p. 116-117.
} 
En d'autres termes, par un geste dans lequel nous nous inscrivons sans réserve, Gallagher a récolté les fruits du problème de la naturalisation de l'husserlianisme que nous entrevoyons dans l'enrichissement zahavien de la nature et la nécessité de "phénoménologiser » celle-ci, afin, selon un célèbre vocable hégélien, d'ouvrir une nouvelle figure de la question de la naturalisation de la phénoménologie depuis la nature et dans un dialogue avec la physique contemporaine. Or un tel dialogue nous semble encouragé par la physique elle-même plutôt qu'autre chose : en plus des éléments discutés par l'article de Gallagher lui-même, on mentionnera les conclusions tirées de ses lectures de la physique contemporaine par Étienne Klein, et en particulier celle qui nous indique qu' " une particule quantique n'est pas un corpuscule ${ }^{1}$ », qu'en physique quantique, "les notions mêmes d'objet et de trajectoire $[\ldots]$ deviennent problématiques ${ }^{2} »$, ce qui conduit Klein à conclure à une " matière $[\ldots]$ déchosifiée $e^{3} »$, laquelle nous semble renforcer la nécessité de penser une physis comme «anti-Nature». Dans cette perspective, on rapportera également que, selon Klein, « la notion de cause, au sens fort du terme, a posé tant de problèmes aux physiciens qu'ils ont fini par quasiment l'abandonner ${ }^{4} »$. Qui plus est, à propos de "l'intrication quantique ${ }^{5}$ », Klein conclut qu' " en physique quantique, la connaissance des parties, si loin qu'elle soit poussée, semble insuffisante à fournir une connaissance du tout ${ }^{6} »$, ce qui l'amène à préciser :

La paire formée par les deux particules a des propriétés globales que n'ont pas les particules individuelles. En somme, le tout qu'elle forment d'une part n'a pas de localisation précise, d'autre part est plus que l'ensemble de ses parties ${ }^{7}$.

Ces principes issus de la physique contemporaine ont à l'évidence une portée pour la tentative de constitution d'une physis comme "anti-Nature » que nous voulons penser au titre de l'ensemble des «pré-conditions» de la phénoménologie. En effet, l'abandon de la cause et les conclusions tirées par

${ }^{1}$ Étienne Klein (2018). Matière à contredire. Essai de philo-physique. Paris : Les éditions de l'Observatoire, p. 155 (note 1).

${ }^{2}$ Étienne Klein (2016). Petit voyage dans le monde des quanta. Paris : Flammarion, p. 11.

${ }^{3}$ Étienne Klein (2018). Op. cit., p.139.

${ }^{4}$ Ibid., p. 109.

${ }^{5}$ Voir, Klein (2004). Op. cit., ch. 6.

${ }^{6}$ Ibid., p. 93.

${ }^{7}$ Étienne Klein (2018). Op. cit., p. 162. 
Klein de "l'intrication quantique » permettent bien d'esquisser une physis non vitale comme "négation même de toutes les dimensions de l'idée classique de Nature» et semblent bien abonder dans le sens du rejet gallagherien du partes extra partes ainsi que dans le sens du concept ruyérien de «surfaces absolues » et leur essentielle «dé-localisation » ${ }^{1}$. Il ne s'agit cependant bien là, pour le moment, que d'esquisses, et il nous paraît de ce fait essentiel d'indiquer plus précisément quelle direction philosophique devra prendre la nouvelle figure de la naturalisation de l'husserlianisme que nous décrivons ${ }^{2}$.

\footnotetext{
${ }^{1}$ Raymond Ruyer, L'animal, l'homme, la fonction symbolique, op. cit., p. 84. Les possibilités d'articulation du ruyérisme avec la physique contemporaine ont été plusieurs fois soulignées, notamment par Élie During dont on pourra consulter l'article qui insiste sur l'importance de la «non-localité » chez Ruyer : Survoler le temps : l'espace-temps comme schème, mythe et thème. Revue de métaphysique et de morale, soumis à la revue. On lira également avec profit le travail de François Brémondy (2007). Ruyer et la physique quantique ou le cadeau royal de la physique contemporaine à la philosophie ». Les études philosophiques, 1 (80), p. 39-62, et notamment ses remarques sur l'importance de la "physique contemporaine » pour le ruyérisme et son concept de «survol». On soulignera enfin que ce qu'Étienne Klein a récemment nommé "philo-physique» (2018, op. cit., sous-titre) n'est pas sans rappeler la « Philosophie-Science » de Ruyer lui-même : (2007). Raymond Ruyer par lui-même. Les Études philosophiques, 1 (80), p. 3-14.

${ }^{2}$ Lors d'une Summer School du Center for Subjectivity Research de Copenhague à laquelle nous avions assisté, Galen Strawson, panpsychiste contemporain, remarquait en répondant aux questions après sa conférence que sa compréhension de la «conscience» n'était à son avis pas incompatible avec le "pré-réflexif» des phénoménologues et en particulier avec celui de Dan Zahavi. Une telle suggestion n'est pas sans importance pour nos préoccupations présentes et mériterait indéniablement d'être étayée, nous ignorons si tel a été le cas depuis l'événement en question. Par ailleurs, dans son chapitre de 2010, Dan Zahavi remarquait dans une note qu'«il devrait être remarqué que certains prétendent que les pleines implications théoriques de la révolution en physique d'Einstein et Bohr doivent encore être réalisées. » S'appuyant sur Ryckmann, Zahavi indique alors qu'Herman Weyl, décisif pour « la théorie de la relativité générale et le champ de la mécanique quantique, non seulement s'est abondamment servi de la critique du naturalisme de Husserl, mais était également profondément influencés par l'idéalisme transcendantal de Husserl ». Zahavi conclut alors indiquant que "certains développements décisifs en physique théorique » ne semblent pas incompatibles avec le projet phénoménologique en général, p. 17.
} 


\section{Conclusion : vers un naturalisme enrichi}

Une relecture ruyérienne du problème de la naturalisation de l'husserlianisme paraît ainsi engageante à plus d'un titre, en particulier parce qu'elle laisse entrevoir l'exacte convergence philosophique des consciences phénoménale et d'accès, non plus dans une "Vie ", mais bien dans une physis nonobjectale; une telle convergence dans une nature qui ne soit pas toujoursdéjà vitalisée par «l'empire » de la «Vie» nous semblant en effet être la seule possibilité pour la phénoménologie d'achever l'enquête sur ses "préconditions ». Un tel projet que nous ne pouvons préciser plus avant ici et qui nous guidera à l'avenir devra cependant faire face à deux difficultés importantes que nous ne pouvons éluder. En effet, nous avons montré que Ruyer offre la possibilité de penser un naturel en un sens phénoménologisé, mais ce naturel ne peut être considéré comme phénoménologisé, pour le moment, qu'en un sens uniquement :

Premièrement, Colonna a déjà montré que «quant à la phénoménologie, Ruyer en est fort distant [...]. Elle est [selon lui] dénuée de sens cosmique [...]. Elle ne tient jamais compte du fait que l'homme percevant possède un cerveau et un système nerveux ", ainsi, "l'intentionnalité risque [...] de flotter dans une région à part, sans jamais toucher le réel ${ }^{1} »$. Deuxièmement, si la conscience phénoménale peut cesser de nous obséder avec Ruyer, sa capacité d'accès est beaucoup plus problématique. Barbaras a en effet séjourné suffisamment longtemps dans le ruyérisme pour l'écarter définitivement, constatant, chez Ruyer, "le sacrifice pur et simple de la dimension intentionnelle de la conscience et, partant, l'incapacité de rendre compte de manière satisfaisante de la conscience perceptive ${ }^{2}$. $"$ Pour le moment, la physis ne peut donc être considérée comme enrichie que partiellement: si la conscience ruyérienne semble permettre d'enrichir le cognitif cérébral et plus largement le naturel, c'est pour le moment uniquement au moyen de sa dimension phénoménale (au sens de Block), autrement dit, le cognitif est enrichi d'une conscience qui n'est pas encore en même temps la conscience de quelque chose des phénoménologues.

En d'autres termes, la relecture ruyérienne de la naturalisation de l'husserlianisme que nous proposons, si elle peut parfaitement prétendre se

${ }^{1}$ Fabrice Colonna, «Un panpsychiste dans le siècle » entretien avec Élie During publié dans (2014). Critique, LXX (804), p. 436. Ainsi, « entre Ruyer et MerleauPonty l'échange confine au dialogue de sourds ». Fabrice Colonna (2014). MerleauPonty et le renouvellement de la métaphysique. Paris : Hermann, p. 288.

${ }^{2} I P V$, p. 180. 
défaire de l'accusation selon laquelle la phénoménologie ne prendrait pas en compte le système nerveux central de l'être humain, devra anticiper le risque philosophique majeur que constitue le sacrifice de l'intentionnalité. Nous avons cependant de bonnes raisons de penser qu'un tel problème peut être pallié. En effet, le sacrifice déploré par Barbaras nous semble étayé sur une lecture partiellement injuste de l'œuvre ruyérienne : ce fait critiqué par le phénoménologue repose sur une lecture du Néo-finalisme à l'aune de travaux plus anciens que ce dernier et pour lesquels cette critique est vraisemblablement valide ${ }^{1}$. Pour autant, il nous semble que la situation est différente dans Néo-finalisme et qu'elle autorise de ce fait une lecture divergente. Le problème pointé par Barbaras n'en disparaîtrait pas pour autant, mais pourrait être rediscuté, avec, à terme, la possibilité de penser un cognitif participant d'une physis enrichie d'une authentique conscience intentionnelle.

En définitive, si la question de la naturalisation de l'husserlianisme veut trouver sa réponse dans un enrichissement et une phénoménologisation de la nature, elle devra se confronter à l'immense effort philosophique ruyérien ${ }^{2}$. Si la phénoménologie tient à s'assimiler la nature, ce ne sera qu'à la condition d'intégrer dans son déploiement le cognitif, le neuronal et plus largement le naturel comme autant de ses "pré-conditions». En nous donnant les moyens naturalistes non plus d'une « refonte du hard problem » mais bien du constat de sa qualité de «problème apparent», en nous proposant une nature enrichie par voie de "formation active " "selon une norme », et non selon la Vie, Ruyer nous promet la perspective d'une phénoménologie articulée au naturel capable d' " ancestralité » et susceptible de penser la non-vie avec la vie. Accompagnée de Ruyer, il semble que la

\footnotetext{
${ }^{1} I P V$, deuxième partie, premier chapitre.

${ }^{2}$ Nous signalons que nous sommes redevable des excellents cours d'André Conrad sur Néo-finalisme, lequel signalait entre autres ses doutes quant à l'impossible pensée de l'intentionnalité chez Ruyer pointée par Barbaras et la pertinence du ruyérisme pour la philosophie contemporaine de l'esprit et la philosophie de la physique contemporaine, nous encourageant en ce sens à nous informer du travail d'Étienne Klein. Le lecteur pourra consulter l'efficace Introduction au numéro d'Alter consacré à la nature par Jean-Claude Gens et Grégori Jean, insistant sur les raisons d'une tendance de la phénoménologie à se penser comme « paradigme "antinaturel" "; par exemple, on trouve dans la «tradition française » quelque chose comme « une nouvelle architectonique philosophique fondée sur des instances (l'Autre, la Vie) », laquelle ménage « peu de place à la nature » et lui confère « une place $[. .$.$] secondaire et dérivée ». Jean-Claude Gens et Grégori Jean (2018). Intro-$ duction. Alter, 26, p. 10-11.
} 
phénoménologie trouvera ainsi, en poursuivant sur son chemin naturaliste emprunté ces dernières années, son « sens cosmique ».

Remerciements. Ce texte a bénéficié des relectures, conseils de remaniements et demandes de précisions d'Étienne Bimbenet que nous remercions pour son aide aussi précieuse que déterminante ainsi que pour son soutien et son encadrement en général en tant que co-directeur de thèse ; Benjamin Berger a également relu une version antérieure de ce travail et nous le remercions pour ses remarques. Des versions antérieures de cet article ont été présentées lors de la journée d'étude du séminaire de la revue Alter, aux Archives Husserl de Paris (CNRS - UMR 8547) en décembre 2016, lors du "Copenhagen-Leuven-Paris Workshop» au Center For Subjectivity Research de l'Université de Copenhague en mars 2017, au colloque «Embodiment, Neuroscience and Neuropsychiatry : A Multidisciplinary Conference with Shaun Gallagher » à l'Université de Lausanne en novembre 2017 et enfin dans le cadre de la journée d'étude «Les sciences cognitives et leurs philosophies : cartésianisme, phénoménologie, pragmatisme »de l'Université de Franche-Comté. Nous remercions les organisatrices et organisateurs de ces événements ainsi que les participants pour leurs remarques, critiques et échanges, qui nous ont permis de progresser dans l'écriture de ce texte. Ce texte est également redevable des échanges que nous avons pu avoir avec Rémy Amouroux - dont l'encadrement et le soutien en tant que co-directeur nous ont été essentiels -, Shaun Gallagher, Dan Zahavi, Laurence Kaufmann, Mathieu Arminjon, Ovidiu Stanciu, Françoise Schenk, Élodie Boublil, Marine Kneubühler et Hugues Poltier qui nous ont permis d'enrichir notre compréhension des problèmes qui sont les nôtres. Enfin, nous tenons à adresser des remerciements appuyés à Jean-Michel Roy : nos collaborations diverses, nos échanges fréquents et substantiels, ses conseils de lecture, ses remarques éventuellement critiques, sa considération et son soutien ont été très précieux pour nous et nous ont permis d'affiner et de consolider nos propres conceptions ; ce texte lui est redevable en ce sens. 